J. Clin. Chem. Clin. Biochem.

Vol. 24, 1986, pp. $1001-1007$

(C) 1986 by Walter de Gruyter \& Co. Berlin - New York

\title{
Bestimmung von Enzymaktivitäten in Urinen von Calciumoxalatstein-Patienten
}

\author{
Von J. H. Prinsen, Heidrun Günther ${ }^{1}$ ) und J. Breuer \\ Zentrallaboratorium des Marienhospitals Gelsenkirchen
}

(Eingegangen am 3. Juni/28. August 1986)

Zusammenfassung: Die katalytischen Aktivitäten von Lactatdehydrogenase, $\gamma$-Glutamyltransferase, alkalischer Phosphatase, Alaninaminopeptidase und $\mathrm{N}$-Acetyl- $\beta$ - $D$-glucosaminidase wurden im 24-Stunden-Urin von Calciumoxalatstein-Patienten (9 Männer, 11 Frauen) gemessen und mit denen von einem Normalkollektiv (11 Männer, 10 Frauen) verglichen. Die diagnostische Sensitivität und die diagnostische Spezifität wurden nach vier verschiedenen Diskriminationsverfahren berechnet, wobei die Sensitivität zwischen $65 \%-100 \%$ lag, die Spezifität zwischen $72 \%-100 \%$.

Beim Normalkollektiv war eine deutliche Korrelation in der Ausscheidung der drei Bürstensaumenzyme vorhanden, während bei den Calciumoxalatstein-Patienten nur eine Korrelation zwischen $\gamma$-Glutamyltransferase- und $\mathrm{N}$-Acetyl- $\beta$ - $D$-glucosaminidase-Exkretion nachzuweisen war. Hieraus lassen sich pathologische Schlüsse ziehen.

\section{Determination of catalytic activities in urines of patients with calcium urolithiasis}

Summary: The catalytic activity of lactate dehydrogenase, $\gamma$-glutamyltransferase, alkaline phosphatase, alanine aminopeptidase and $\mathrm{N}$-acetyl- $\beta-D$-glucosaminidase has been measured in $24 \mathrm{~h}$ urines of patients with a calcium oxalate calculus ( 9 men, 11 women) and has been compared with those of a reference collective (11 men, 10 women). The diagnostic sensitivity and the diagnostic specificity have been calculated according to four different discrimination methods in which the diagnostic sensitivity lies between $65 \%$ and $100 \%$, the diagnostic specificity between $72 \%$ and $100 \%$.

Within the reference group there was a correlation between the excretion of the three brush-border enzymes, whereas within the group of patients only a correlation between $\gamma$-glutamyltransferase and $\mathrm{N}$-acetyl- $\beta-D$ glucosaminidase was observed. From this pathophysiological conclusions can be drawn.

\section{Einführung}

In Westeuropa beträgt die Inzidenz des Harnsteinleidens $1-2 \%$ (1). In dieser Hinsicht ist es mit dem Diabetes mellitus und den rheumatischen Erkrankungen vergleichbar.

1) Diese Veröffentlichung enthält wesentliche Teile der Dissertation von Frau Heidrun Günther für die Promotion an der Medizinischen Fakultät dẹr Universität (Gesamthochschule) Essen, 1986.
Unter den Harnsteinen herrscht der Calciumoxalatstein vor. In $65 \%$ bildet dieser Stoff die Hauptsubstanz der vorkommenden Steine (2).

Über den Mechanismus der Harnsteinentstehung in der Niere existiert keine einheitliche Theorie. Am häufigsten genannt werden die Matrix- und Kristallisationstheorie $(3,4)$. Bei diesen beiden Entstehungserklärungen spielen organische Harnbestandteile eine große Rolle.

J. Clin. Chem. Clin. Biochem. / Vol. 24, 1986 / No. 12 
Um zur Diagnostik bei Harnsteinpatienten beizutragen und einen Beitrag zum Verständnis der Harnsteinentstehung zu leisten, haben wir Messungen von Enzymaktivitäten im Urin durchgeführt.

Von 70 mit dem Harn ausgeschiedenen Enzymen (5) wählten wir folgende fünf aus:

- Lactatdehydrogenase (EC 1.1.1.27)

- N-Acetyl- $\beta$-D-glucosaminidase (EC 3.2.1.30)

- $\gamma$-Glutamyltransferase (EC 2.3.2.2)

- Alkalische Phosphatase (EC 3.1.3.1)

- Alaninaminopeptidase (EC 3.4.11.2)

Drei von diesen fünf Enzymen ( $\gamma$-Glutamyltransferase, alkalische Phosphatase und Alaninaminopeptidase) sind größtenteils in der tubulären Zellmembran lokalisiert. Eine Veränderung der Ausscheidung dieser drei Enzyme im Urin kann über mechanische Beschädigung des Tubulus durch den Stein Aufschluß geben.

\section{Methodik und Patientengut}

Es wurden 9 Männer (30-50, Durchschnittsalter 43 Jahre) und 11 Frauen (30-78, Durchschnittsalter 57 Jahre) mit idiopathischer Calciumoxalat-Urolithiasis in die Studie einbezogen. Die Gruppe der Normalpersonen setzt sich aus 11 Männern (1757, Durchschnittsalter 36 Jahre) und 10 Frauen (17-51, Durchschnittsalter 38 Jahre) zusammen.

Zum Zeitpunkt der Enzymaktivitätsmessung waren bei allen Patienten Nierensteine vorhanden. Die Nierensteine wurden nach spontanem Abgang oder operativer Entfernung mit Hilfe der I.R.-Spektroskopie analysiert. Die Steine bestanden aus mindestens $70 \%$ Calciumoxalat (Weddellit, Whewellit) und $\mathrm{zu}$ höchstens $30 \%$ aus Calciumphosphat (Apatit).

15 der 20 Patienten litten zum ersten Mal unter Urolithiasis. Bei den übrigen 5 Patienten lag die vorhergehende Nephrolithiasis mehr als zwei Jahre zurück. Die Personen der Referenzgruppe waren laut Anamnese und Labordiagnostik frei von erkennbaren Krankheiten. Sie standen nicht unter Dauermedikation. Keine dieser Personen hatte - auch ein Jahr nach dieser Studie - einen Nierenstein. Bei keiner der Frauen lag während der Studie eine Gravidität vor.

\section{Sammlung von Urin und Probenvorbereitung}

Die Harnsammlung erfolgte über einen Zeitraum von $24 \mathrm{~h}$ in ein Sammelgefä $B$, das vorher mit $5 \mathrm{ml}$ Thymollösung $(150 \mathrm{~g} / \mathrm{l}$ Isopropanol) zur Konservierung des Harns versetzt worden war. Es wurde von jeder Person eine Urinprobe untersucht.

Bei allen 24-h-Sammelurinen wurde ein Urinstatus (Combur ${ }^{\circledR}$. 9-Test Boehringer Mannheim und mikroskopische Untersuchung) durchgeführt, wobei sowohl bei den Kontrollpersonen als auch bei der Calciumoxalatsteingruppe kein pathologischer Befund zu erheben war. Vor der weiteren Aufbereitung wurden die Proben $10 \mathrm{~min}$ lang bei $1000 \mathrm{~g}$ zentrifugiert. Anschließend wurde der Überstand an Separating Columns (Roche Diagnostica Nr. 0791768) nach Maruhn (16) gelfiltriert. Gleich danach erfolgte die komplette Enzymaktivitätsmessung oder aber die Urine wurden sofort nach Gelfiltration bei $-20^{\circ} \mathrm{C}$ eingefroren, und die Messung fand innerhalb von 3 Tagen statt. Im letzteren Fall haben wir für die Lactatdehydrogenase-Messung einen Teil des gelfiltrierten Urins bei +2 bis $+8^{\circ} \mathrm{C}$ aufbewahrt. Die Kreatininbestimmung im Urin wurde stets im Anschluß an die Harnsammlung durchgeführt.

\section{MeBmethoden}

Die Enzymaktivitätsmessung erfolgte bei $25^{\circ} \mathrm{C}$. Lactatdehydrogenase, alkalische Phosphatase, $\gamma$-Glutamyltransferase und Alaninaminopeptidase wurden am Gemsaec-Zentrifugal-Analyser der Firma E.N. I. durchgeführt, während N-Acetyl- $\beta$ - $D$ glucosaminidase mit Hilfe des Eppendorf-Enzymrechners Nr. CKE 6455 bestimmt wurde.

Die Endkonzentration im Reaktionsgemisch war für:

- $\gamma$-Glutamyltransferase $(7,8)$

$100 \mathrm{mmol} / \mathrm{l}$ Trispuffer $\mathrm{pH}=8.5$

$100 \mathrm{mmol} / \mathrm{l}$ Glycylglycin und

$4 \mathrm{mmol} / 1 \mathrm{\gamma}$-Glutamyl-3-carboxy-4-nitroanilid

- Alaninaminopeptidase (10)

$0,1 \mathrm{~mol} / 1$ Phosphatpuffer $\mathrm{pH}=7.6$

$1,7 \mathrm{mmol} / 1$ L-Alanin-p-nitroanilid

- Lactatdehydrogenase (6)

$0,6 \mathrm{mmol} / 1$ Natriumpyruvat

$0,18 \mathrm{mmol} / 1 \mathrm{NADH}$

$50,0 \mathrm{mmol} / 1$ Phosphatpuffer $\mathrm{pH}=7.5$

- Alkalische Phosphatase (9)

$1,0 \mathrm{~mol} / 1$ Diethanolalanin-HCl-Puffer $\mathrm{pH}=9.8$

$0,5 \mathrm{mmol} / 1$ Magnesiumchlorid

$10 \mathrm{mmol} / 1$ 4-Nitrophenylphosphat

- N-Acetyl- $\beta-D$-glucosaminidase (11)

$0,25 \mathrm{~mol} / 1$ 2-Amino-2-methyl-1-propanol-HCl=Puffer 3,3 mmol/1 4-Nitrophenyl- $\mathrm{N}$-acetyl- $\beta$ - $D$-glucosaminid $0,33 \mathrm{mmol} / 1$ Citratpuffer $\mathrm{pH}=4.15$

Inkubationstemperatur: $37^{\circ} \mathrm{C}$

Bei der Messung von Lactatdehydrogenase, alkalischer Phosphatase und $\gamma$-Glutamyltransferase wurde nach der optimierten Standard-Methode der Deutschen Gesellschaft für Klinische Chemie verfahren.

Da die Enzymaktivitäten von alkalischer Phosphatase und Lactatdehydrogenase im Urin meist relativ niedrig sind, wurde die eingesetzte Urinmenge im Falle der Lactatdehydrogenase auf das 12,5fache und bei alkalischer Phosphatase auf das 25fache bei Beibehaltung der vorgeschriebenen Endkonzentration der Reagenzien erhöht. Alaninaminopeptidase wurde nach der $\mathrm{Me}-$ thode von Mondorf (10) gemessen und $\mathrm{N}$-Acetyl- $\beta$ - $D$-glucosaminidase nach Maruhn (11).

Die Bestimmung der Kreatininkonzentration in den nicht gelfiltrierten Urinen wurde bei $37^{\circ} \mathrm{C}$ am ASTRA 4 der Firma Beckman ausgeführt und beruhte auf einer modifizierten JaffeReaktion.

Die Reagenzien wurden mit Ausnahme von 4-Nitrophenyl-Nacetyl- $\beta$ - $D$-glucosaminid (Firma Calbiochem) von der Firma E. Merck, Darmstadt, bezogen.

\section{Statistische Berechnung}

Die Bearbeitung der Analysenergebnisse wurde nach dem Diskriminationsverfahren - entwickelt durch Dr. E. Hansert (24) - ausgeführt.

\section{Resultate}

Die Analysenergebnisse der Calciumoxalatstein-Patienten sind in Tabelle 1, die der Kontrollgruppe in Tabelle 2 wiedergegeben.

Tabelle 3 zeigt die Medianwerte und Streubreite beider Gruppen. 
Tab. 1. Einzelergebnisse der Aktivitätsbestimmungen von fünf Enzymen in den Urinen von 20 Calciumoxalatstcin-Patienten (9 Männer, 11 Frauen). Einheit: U/mol Kreatinin.

\begin{tabular}{|c|c|c|c|c|c|c|}
\hline & $\begin{array}{l}\text { Alter } \\
\text { (a) }\end{array}$ & $\begin{array}{l}\gamma \text {-Glutamyl- } \\
\text { transferase }\end{array}$ & $\begin{array}{l}\mathrm{N} \text {-Acetyl- } \beta-D \text { - } \\
\text { glucosaminidase }\end{array}$ & $\begin{array}{l}\text { Alkalische } \\
\text { Phosphatase }\end{array}$ & $\begin{array}{l}\text { Lactatdehy- } \\
\text { drogenase }\end{array}$ & $\begin{array}{l}\text { Alaninamino- } \\
\text { peptidase }\end{array}$ \\
\hline \multicolumn{7}{|c|}{ Männer } \\
\hline $\begin{array}{l}\text { P.H. } \\
\text { K.M. } \\
\text { F.M. } \\
\text { L.O. } \\
\text { J.U. } \\
\text { S.M. } \\
\text { U.S. } \\
\text { K.S. } \\
\text { W.R. }\end{array}$ & $\begin{array}{l}33 \\
34 \\
37 \\
44 \\
45 \\
45 \\
47 \\
50 \\
50\end{array}$ & $\begin{array}{r}1993 \\
869 \\
1721 \\
1199 \\
1720 \\
1686 \\
1322 \\
4988 \\
1835\end{array}$ & $\begin{array}{r}688 \\
151 \\
161 \\
0 \\
426 \\
412 \\
621 \\
873 \\
1129\end{array}$ & $\begin{array}{l}280 \\
164 \\
331 \\
143 \\
537 \\
249 \\
430 \\
516 \\
374\end{array}$ & $\begin{array}{r}46 \\
144 \\
78 \\
61 \\
61 \\
225 \\
797 \\
1505 \\
534\end{array}$ & $\begin{array}{l}127 \\
185 \\
123 \\
154 \\
171 \\
233 \\
143 \\
368 \\
344\end{array}$ \\
\hline \multicolumn{7}{|c|}{ Frauen } \\
\hline $\begin{array}{l}\text { K.W. } \\
\text { J.S. } \\
\text { P.W. } \\
\text { E.W. } \\
\text { S. K. } \\
\text { G.H. } \\
\text { L.H. } \\
\text { R.H. } \\
\text { C. B. } \\
\text { C.S. } \\
\text { M.B. }\end{array}$ & $\begin{array}{l}30 \\
44 \\
44 \\
48 \\
57 \\
63 \\
63 \\
64 \\
70 \\
71 \\
78\end{array}$ & $\begin{array}{r}3507 \\
2538 \\
6760 \\
1554 \\
2221 \\
2769 \\
2262 \\
213 \\
1328 \\
2984 \\
1497\end{array}$ & $\begin{array}{r}1689 \\
547 \\
4431 \\
314 \\
1193 \\
138 \\
588 \\
43 \\
458 \\
684 \\
511\end{array}$ & $\begin{array}{r}1169 \\
2612 \\
1980 \\
402 \\
713 \\
443 \\
433 \\
405 \\
767 \\
649 \\
14\end{array}$ & $\begin{array}{r}606 \\
482 \\
43538 \\
3369 \\
1768 \\
235 \\
855 \\
277 \\
897 \\
177 \\
119\end{array}$ & $\begin{array}{r}606 \\
155 \\
215 \\
314 \\
343 \\
526 \\
588 \\
0 \\
112 \\
189 \\
105\end{array}$ \\
\hline
\end{tabular}

Tab. 2. Einzelergebnisse der Aktivitätsbestimmungen von fünf Enzymen in den Urinen von 21 Normalpersonen (11 Männer, 10 Frauen). Einheit: U/mol Kreatinin.

\begin{tabular}{|c|c|c|c|c|c|c|}
\hline & $\begin{array}{l}\text { Alter } \\
\text { (a) }\end{array}$ & $\begin{array}{l}\gamma \text {-Glutamyl- } \\
\text { transferase }\end{array}$ & $\begin{array}{l}\text { N-Acetyl- } \beta-D \text { - } \\
\text { glucosaminidase }\end{array}$ & $\begin{array}{l}\text { Alkalische } \\
\text { Phosphatase }\end{array}$ & $\begin{array}{l}\text { Lactatdehy- } \\
\text { drogenase }\end{array}$ & $\begin{array}{l}\text { Alaninamino- } \\
\text { peptidase }\end{array}$ \\
\hline \multicolumn{7}{|c|}{ Männer } \\
\hline $\begin{array}{l}\text { K.U. } \\
\text { S.M. } \\
\text { M.R. } \\
\text { U.J. } \\
\text { T.R. } \\
\text { J.P. } \\
\text { M.J. } \\
\text { R.S. } \\
\text { J.B. } \\
\text { W.G. } \\
\text { K.R. }\end{array}$ & $\begin{array}{l}17 \\
18 \\
22 \\
23 \\
25 \\
31 \\
47 \\
48 \\
52 \\
54 \\
57\end{array}$ & $\begin{array}{r}2248 \\
1409 \\
1144 \\
1105 \\
813 \\
1028 \\
1537 \\
1696 \\
1355 \\
2459 \\
703\end{array}$ & $\begin{array}{r}182 \\
218 \\
247 \\
238 \\
244 \\
211 \\
201 \\
461 \\
266 \\
1130 \\
378\end{array}$ & $\begin{array}{l}272 \\
266 \\
155 \\
226 \\
211 \\
132 \\
370 \\
333 \\
154 \\
718 \\
119\end{array}$ & $\begin{array}{r}300 \\
304 \\
99 \\
31 \\
211 \\
274 \\
467 \\
224 \\
83 \\
292 \\
270\end{array}$ & $\begin{array}{l}258 \\
144 \\
148 \\
116 \\
130 \\
171 \\
195 \\
243 \\
106 \\
306 \\
113\end{array}$ \\
\hline \multicolumn{7}{|c|}{ Frauen } \\
\hline $\begin{array}{l}\text { D.R. } \\
\text { J.S. } \\
\text { H.G. } \\
\text { M.G. } \\
\text { C.S. } \\
\text { A. K. } \\
\text { K.P. } \\
\text { M.G. } \\
\text { L.J. } \\
\text { C.R. }\end{array}$ & $\begin{array}{l}17 \\
21 \\
22 \\
45 \\
45 \\
47 \\
48 \\
48 \\
48 \\
51\end{array}$ & $\begin{array}{l}1103 \\
2122 \\
1460 \\
2179 \\
1533 \\
1621 \\
1854 \\
2332 \\
1270 \\
1065\end{array}$ & $\begin{array}{l}287 \\
535 \\
390 \\
438 \\
327 \\
291 \\
344 \\
830 \\
410 \\
414\end{array}$ & $\begin{array}{l}147 \\
628 \\
218 \\
504 \\
411 \\
131 \\
452 \\
830 \\
339 \\
304\end{array}$ & $\begin{array}{r}198 \\
543 \\
45 \\
856 \\
126 \\
10 \\
280 \\
840 \\
268 \\
296\end{array}$ & $\begin{array}{r}176 \\
178 \\
82 \\
219 \\
193 \\
225 \\
154 \\
241 \\
95 \\
118\end{array}$ \\
\hline
\end{tabular}

Die diagnostischen Entscheidungsgrenzen wurden so festgesetzt, daß die größtmögliche Effizienz erzielt wurde ${ }^{2}$ ). Aus der Tabelle 4 können die optimalen ein-

\footnotetext{
2) Herrn Dr. E. Hansert, München, danken wir für die Berechnung der Daten.
}

dimensionalen Entscheidungspunkte für die MeßgröBen bei Frauen bzw. Männern entnommen werden. So wird bei Frauen eine Spezifität von $80 \%$ und eine Sensitivität von $82 \%$ erreicht. Diese Berechnung legt zugrunde, daß Personen dann als Calciumoxalatstein- 
Tab. 3. Mediane und Meßbereiche der katalytischen Aktivitäten von fünf Enzymen in den Urinen von Normalpersonen und Calciumoxalatstein-Patienten. Einheit: U/mol Kreatinin.

\begin{tabular}{|c|c|c|c|c|c|c|}
\hline \multirow{2}{*}{$\overline{\gamma \text {-Glutamyltransferase }}$} & & \multicolumn{2}{|c|}{ Referenzgruppe } & \multicolumn{3}{|c|}{ Calciumoxalatstein-Patienten } \\
\hline & & Mediane & Meßbereiche & Mediane & & Meßbereiche \\
\hline & $\begin{array}{l}9 \\
0 \\
0\end{array}$ & $\begin{array}{l}1577 \\
1250\end{array}$ & $\begin{array}{r}1065-2332 \\
703-2459\end{array}$ & $\begin{array}{l}2262 \\
1720\end{array}$ & .1 & $\begin{array}{l}213-6760 \\
869-4988\end{array}$ \\
\hline $\mathrm{N}$-Acetyl- $\beta$ - $D$-glucosami & $\begin{array}{l}9 \\
8\end{array}$ & $\begin{array}{l}400 \\
244\end{array}$ & $\begin{array}{l}287-830 \\
183-1130\end{array}$ & $\begin{array}{l}547 \\
426\end{array}$ & & $\begin{array}{r}43-4431 \\
0-1129\end{array}$ \\
\hline Alkalische Phosphatase & $\begin{array}{l}q \\
\vdots\end{array}$ & $\begin{array}{l}375 \\
226\end{array}$ & $\begin{array}{l}131-830 \\
119-718\end{array}$ & $\begin{array}{l}649 \\
331\end{array}$ & & $\begin{array}{r}14-2612 \\
143-\quad 537\end{array}$ \\
\hline Lactatdehydrogenase & $\begin{array}{l}q \\
\sigma\end{array}$ & $\begin{array}{l}274 \\
270\end{array}$ & $\begin{array}{l}10-856 \\
31-467\end{array}$ & $\begin{array}{l}606 \\
144\end{array}$ & & $\begin{array}{r}119-43538 \\
46-1505\end{array}$ \\
\hline Alaninaminopeptidase & $\begin{array}{l}q \\
0 \\
0\end{array}$ & $\begin{array}{l}177 \\
148\end{array}$ & $\begin{array}{r}82-241 \\
106-306\end{array}$ & $\begin{array}{l}215 \\
171\end{array}$ & & $\begin{array}{r}0-606 \\
120-\quad 368\end{array}$ \\
\hline
\end{tabular}

Tab. 4. Optimale Entscheidungsgrenzen für die Einzelmeßgrößen bei Frauen bzw. Mä̀nnern. Meßergebnisse, die über der optimalen Grenze liegen, werden als positiv (krank) eingeordnet.

\begin{tabular}{|c|c|c|c|c|c|}
\hline Enzym & & $\begin{array}{l}\text { Optimale Grenze } \\
\text { U/mol } \\
\text { Kreatinin }\end{array}$ & $\begin{array}{l}\text { Sensitivität } \\
(\%)\end{array}$ & $\begin{array}{l}\text { Spezifität } \\
(\%)\end{array}$ & $\begin{array}{l}\text { Effizienz } \\
(\%)\end{array}$ \\
\hline \multicolumn{6}{|c|}{$\gamma$-Glutamyltransferase } \\
\hline & $\begin{array}{l}q \\
\vdots \\
0\end{array}$ & $\begin{array}{l}2200 \\
1600\end{array}$ & $\begin{array}{l}63,6 \\
66,7\end{array}$ & $\begin{array}{l}90,0 \\
72,7\end{array}$ & $\begin{array}{l}153,6 \\
139,4\end{array}$ \\
\hline \multicolumn{6}{|c|}{$\mathrm{N}$-Acetyl- $\beta$ - $D$-glucosaminidase } \\
\hline & $\begin{array}{l}q \\
0 \\
0\end{array}$ & $\begin{array}{l}540 \\
400\end{array}$ & $\begin{array}{l}54,6 \\
66,7\end{array}$ & $\begin{array}{l}90,0 \\
81,8\end{array}$ & $\begin{array}{l}144,6 \\
148,5\end{array}$ \\
\hline \multicolumn{6}{|c|}{ Alkalische Phosphatase } \\
\hline & $\begin{array}{l}q \\
0\end{array}$ & $\begin{array}{l}640 \\
370\end{array}$ & $\begin{array}{l}54,6 \\
44,4\end{array}$ & $\begin{array}{l}90,0 \\
90,9\end{array}$ & $\begin{array}{l}144,6 \\
135,4\end{array}$ \\
\hline \multicolumn{6}{|c|}{ Lactatdehydrogenase } \\
\hline & $\begin{array}{l}q \\
0\end{array}$ & $\begin{array}{l}600 \\
500\end{array}$ & $\begin{array}{l}54,6 \\
33,3\end{array}$ & $\begin{array}{r}80,0 \\
100,0\end{array}$ & $\begin{array}{l}134,7 \\
133,3\end{array}$ \\
\hline \multicolumn{6}{|c|}{ Alaninaminopeptidase } \\
\hline & $\begin{array}{l}q \\
\delta\end{array}$ & $\begin{array}{l}250 \\
160\end{array}$ & $\begin{array}{l}45,4 \\
66,6\end{array}$ & $\begin{array}{r}100,0 \\
54,5\end{array}$ & $\begin{array}{l}145,4 \\
120,1\end{array}$ \\
\hline
\end{tabular}

Patienten eingestuft werden, wenn mindestens zwei der fünf Werte die Entscheidungsgrenze überschreiten. Bei Männern ist die entsprechende Spezifität $82 \%$, die Sensitivität $67 \%$. Bei diesem Verfahren sind im Gegensatz zu der unten aufgeführten Berechnungsart die Entscheidungsgrenzen so gelegt worden, daß für jedes einzelne Enzym die optimale Effizienz erreicht wird.
In der Tabelle 5 sind die optimalen fünfdimensionalen Entscheidungsgrēnz̄en für die Kombination aller fünf Enzyme aufgeführt. Bei dieser rein mathematischen Problemlösung erhält man bei Frauen eine Sensitivität vón $82 \%$ und eine Spezifität von $100 \%$, bei Männern eine Sensitivität vọn $100 \%$ und eine Spezifität von $72 \%$. 
Tab. 5. Optimaler fünfdimensionaler Entscheidungspunkt für die Kombination aller fünf im Urin von 21 Normalpersonen (10 Frauen, 11 Männer) und 20 Calciumoxalatstein-Patienten (11 Frauen, 9 Männer) bestimmten Enzyme. Die Úberschreitung von mindestens 3 Werten in die angegebene Richtung führt zu Einordnung als positiv (krank).

\begin{tabular}{lcc}
\hline Enzym & \multicolumn{2}{l}{ optimale Grenze } \\
& \multicolumn{2}{c}{ (U/mol Kreatinin) } \\
\hline & Frauen & Männer \\
$\gamma$-Glutamyltransferase & $\geqslant 2540$ & $\geqslant 870$ \\
N-Acetyl- $\beta$ - $D$-glucosaminidase & $\geqslant 460$ & $\geqslant 380$ \\
Alkalische Phosphatase & $\geqslant 400$ & $\geqslant 370$ \\
Lactatdehydrogenase & $\geqslant 860$ & $\geqslant 220$ \\
Alaninaminopeptidase & $\geqslant 310$ & $\geqslant 120$ \\
& & \\
Sensitivität & $81,8 \%$ & $100,0 \%$ \\
Spezifität & $100,0 \%$ & $72,7 \%$ \\
Effizienz & $181,8 \%$ & $172,7 \%$ \\
\hline
\end{tabular}

\section{Diskussion}

Wie wir oben gezeigt haben und im folgenden ausgeführt wird, können mit Hilfe der Enzymaktivitätsbestimmung von alkalischer Phosphatase, Alaninaminopeptidase, $\gamma$-Glutamyltransferase, Lactatdehydrogenase und $\mathrm{N}$-Acetyl- $\beta$ - $D$-glucosaminidase im Urin gesunde Personen von Calciumoxalatstein-Patienten mit einer relativ hohen Sensitivität und Spezifität unterschieden werden.

Der Urin ist im Vergleich zum Serum ein ungünstiges Milieu für die Stabilität der Enzyme. Hinzu kommt die Beeinflussung der Enzymaktivitätsbestimmung durch im Urin vorkommende Inhibitoren. Wie Mattenheimer et al. z. B. für Alaninaminopeptidase gezeigt haben, besteht ein Teil der Inhibitoren aus Aminosäuren (28). Diese beiden Probleme haben wir dadurch gelöst, daß wir einerseits die Inhibitoren durch Gelfiltration eliminiert haben $(15,16)$ und andererseits durch Messung der katalytischen Aktivitäten unmittelbar nach der Sammlung bzw. nach Einfrieren bis zur Messung. Trotzdem fanden wir eine große interindividuelle Variation sowohl bei Normalpersonen als auch bei Calciumoxalatstein-Patienten, jedoch stimmen die Mediane von $\gamma$-Glutamyltransferase, Lactatdehydrogenase, $\quad \mathrm{N}$-Acetyl- $\beta-D$-glucosaminidase, alkalischer Phosphatase und Alaninaminopeptidase in der Referenzgruppe gut mit den in der Literatur angegebenen Werten für Normalpersonen überein $(10,12-23)$.

Die Streuung der Meßwerte ist dafür maßgeblich, daß die Sensitivität der einzelnen Enzymbestimmungen nicht besonders hoch ist. Stuft man Personen dann als Calciumoxalatstein-Patienten ein, wenn mindestens 2 der 5 Enzymwerte über der eindimensionalen Entscheidungsgrenze liegen, erreicht man eine wesentlich höhere diagnostische Effizienz (Tab. 4).

Vergleichbare Ergebnisse erreicht man bei Anwendung der optimalen fünfdimensionalen Entscheidungsgrenzen (Tab. 5), wobei das erstere praxisnäher ist. Die aufgeführten Überlegungen sind nur deshalb notwendig, um die geringfügigen Unterschiede zwischen dem Referenzkollektiv und dem der Patientengruppe deutlicher zu machen, als das bei Betrachtung der einzelnen Enzyme möglich ist.

Von den untersuchten Enzymen sind $\gamma$-Glutamyltransferase, alkalische Phosphatase und Alaninaminopeptidase Bürstensaumenzyme, die Lactatdehydrogenase ein cytoplasmatisches und die N-Acetyl- $\beta-D$ glucosaminidase ein lysosomales Enzym. Um diese Tatsache verwerten zu können, haben wir die gemessenen Enzymaktivitäten einer Spearmanschen Rangkorrelationskoeffizientberechnung (25) unterworfen. Diese gibt Aufschluß über die Größe des statistischen Zusammenhangs der verschiedenen Enzyme. Die Ergebnisse der Berechnung des Korrelationskoeffizienten $r_{s}$ und $\alpha$ für die zehn möglichen Enzymkombinationen sind in Tabelle 6 aufgeführt. Elimination der

Tab. 6. Rangkorrelationskoeffizient $r_{s}$ nach Spearman für die zehn Enzymkombinationen, berechnet aus den Werten der Referenzgruppe bzw. der Calciumoxalatsteingruppe. Das Signifikanzniveau $2 \alpha$ ist nur für die Kombinationen angegeben, bei denen dér Rangkorrelationskoeffizient über 0,70 liegt.

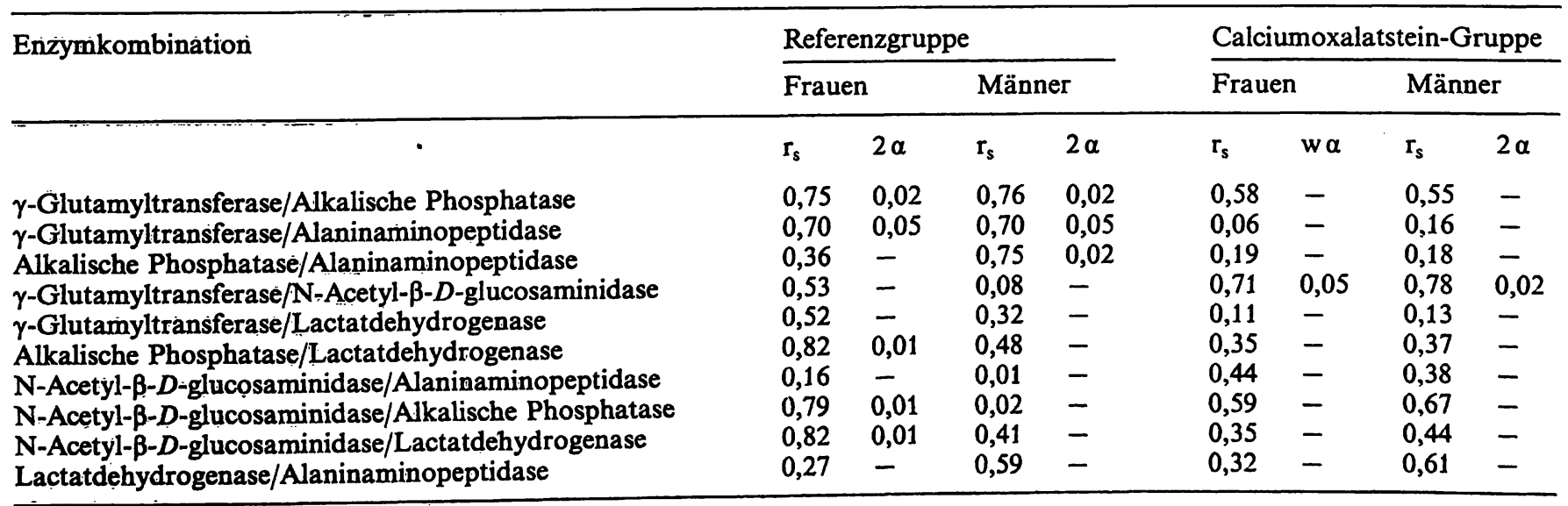


Meßwerte einer weiblichen Kontrollperson mit deutlich statistisch abweichendem AlaninaminopeptidaseWert würde den Rangkorrelationskoeffizienten für alkalische Phosphatase/Alaninaminopeptidase auf 0,72 erhöhen und für Lactatdehydrogenase/Alaninaminopeptidase auf 0,62 , während sie für die übrigen Enzymkombinationen im wesentlichen unverändert blieben. Aus den obengenannten Resultaten kann man bei den Frauen aus der Referenzgruppe eine Korrelation zwischen den Bürstensaumenzymen und den beiden anderen Enzymen erkennen. Das spricht für einen vollständigen Zelluntergang durch natürliche Zellmauserung. Diese Vermutung hat Mattenheimer (26) schon früher geäußert, allerdings hielt er sie für geschlechtsunabhängig. In unserer Studie ergaben die Korrelationsberechnungen bei den männlichen Referenzpersonen nur innerhalb der Bürstensaumenzyme gute Resultate.

Bei den Nephrolithiasis-Patienten ist die Korrelation zwischen den verschiedenen Bürstensaumenzymen total verschwunden. Bei beiden Geschlechtern korrelieren nur $\gamma$-Glutamyltransferase und N-Acetyl- $\beta-D$ glucosaminidase sehr gut. Dieses deutet darauf hin, daß bei den Steinpatienten wahrscheinlich ein Zellschaden im Tubulusapparat vorliegt.

Auch Baggio et al. (21) fanden bei Calciumoxalatstein-Patienten sowohl in Anwesenheit als auch in Abwesenheit eines Steines eine erhöhte Exkretion dieser beiden Enzyme (39), allerdings hat er keine Korrelationsberechnung durchgeführt.

Wenn man die Exkretion und Korrelation von $\gamma$ Glutamyltransferase und Alaninaminopeptidase betrachtet, ist festzustellen, daß die Erklärung dieses Zellschadens nicht nur die mechanische Wirkung des Steines sein kann.

Wenn man davon ausgeht, daß der Stein die Zellmembran mechanisch schädigt, ist $z u$ erwarten, $\mathrm{da} \beta$ die Alaninaminopeptidase-Exkretion prozentual stärker zunimmt als die Ausscheidung der $\gamma$-Glutamyltransferase, da Alaninaminopeptidase sich mehr an der
Außenseite der Bürstensaummembran befindet als $\gamma$ Glutamyltransferase (27): In zehn der zwanzig Fälle der Calciumoxalatgruppe finden wir aber einen $\gamma$ Glutamyltransferase/Alaninaminopeptidase-Koeffizienten größer als zehn trotz einer mechanischen Wirkung des Steines (Referenzwert 6,0-10,0, Tab. 7). Eine mögliche Erklärung hierfür könnte eine primäre Veränderung in der Enzymausscheidung sein, die wiederum ein prädisponierender Faktor für die Steingenese sein kann. Eine Bestätigung dieser Hypothese durch Messung beider Enzyme nach Steinentfernung ist problematisch, da bei zu Rezidiven neigenden Calciumoxalatstein-Patienten stets mit Mikrolithen oder Calciumoxalatkristallen zu rechnen ist.

Tab. 7. Der Koeffizient $\gamma$-Glutamyltransferase/Alaninaminopeptidase der Referenzgruppe und der Calciumoxalatstein-Patienten.

\begin{tabular}{lcccc}
\hline Proband & Frauen & \multicolumn{3}{l}{ Männer } \\
\cline { 2 - 3 } \cline { 5 - 5 } $\begin{array}{l}\text { Referenz- } \\
\text { kollektiv }\end{array}$ & Patienten & $\begin{array}{l}\text { Referenz } \\
\text { kollektiv }\end{array}$ & Patienten \\
\hline 1 & 6,3 & 5,8 & 8,7 & 15,7 \\
2 & 11,9 & 16,4 & 9,8 & 4,7 \\
3 & 17,8 & 31,4 & 7,7 & 14,0 \\
3 & 7,9 & 5,0 & 9,5 & 7,8 \\
5 & 9,9 & 6,5 & 6,3 & 10,1 \\
6 & 7,2 & 5,3 & 6,0 & 7,2 \\
7 & 12,0 & 3,9 & 7,9 & 9,3 \\
8 & 9,7 & 100 & 7,0 & 13,6 \\
9 & 13,4 & 11,9 & 12,8 & 5,3 \\
10 & 9,0 & 15,8 & 8,0 & \\
11 & & 14,3 & 6,3 & \\
Medianwert: & 9,8 & 11,9 & 7,9 & 9,3 \\
\hline
\end{tabular}

Der Koeffizient $\gamma$-Glutamyltransferase/Alaninaminopeptidase ist ein relativ einfacher und praktikabler Diskriminationsfaktor, mit dem als einziges Auswahlkriterium man eine Spezifität von $76 \%$ und eine Sensitivität von $80 \%$ erreicht. Wird daneben noch die Anforderung gestellt, daß eines der beiden Enzyme erhöht sein muß (nach Tab. 4), dann steigt die Spezifität auf $100 \%$, die Sensitivität allerdings fällt auf $65 \%$.

\section{Literatur}

1. Hohenfeller, R. \& Altwein, J. E. (1982) Erkrankungen des Urogenitalsystems, In: Lehrbuch der Inneren Medizin (Gross, R. \& Schölmerich, P., Hrsg.) S. 1118-1120, Schattauer Verlag.

2. Robertson, W. G., Peacock, M., Heyburn, P. J. \& Hanes, F. A. (1980) Scand. J. Urol. Nephrol. 53, 15-28.

3. Hesse, A. \& Bach, D. (1982) Harnsteine: Pathobiochemie und Klinisch-Chemische Diagnostik, Klinische Chemie in Einzeldarstellungen Bd. 5. Thieme Verlag.

4. Hautmann, R. \& Lutzeyer, W. (1980) Der Kalziumoxalatstein, Urolithiasis 3 (Vahlensieck, W., Hrsg.) Springer Verlag.

5. Maruhn, D. \& Paar, D. (1980) Urinary enzymes in therapy monitoring and recognition of side effects of drugs, S. 137-152. Clinical Enzymology Symposia, Verona, Piccin Medical Books, Padua.

6. Empfehlungen der Deutschen Gesellschaft für Klinische Chemie (1972) Standard-Methode zur Bestimmung der Lactat-Dehydrogenase (LDH). J. Clin. Chem. Clin. Biochem. 10, 188-189.

7. Szasz, G., Weimann, G., Stähler, F., Wahlefeld, A.-W. \& Persijn, J. P. (1974) J. Clin. Chem. Clin. Biochem. 12, 228. 
8. Szasz, G. (1974) $\gamma$-Glutamyl-Transpeptidase. In: Methoden der enzymatischen Analyse I (Bergmeyer, H. U., Hrsg.) S. 757-762. Verlag Chemie/Weinheim, Bergstraße.

9. Empfehlungen der Deutschen Gesellschaft für .Klinische Chemie (1970) Standardisierung von Methoden zur Bestimmung von Enzymaktivitäten in biologischen Flüssigkeiten. Z. Klin. Chem. Klin. Biochem. 8, 658.

10. Mondorf, A. W., Breier, J., Hendus, J., Scherberich, J. E., Mackenroth, G., Shah, P. M., Stille, W. \& Schoeppe, W. (1978) Europ. J. Clin. Pharmacol. 13, 133-142.

11. Maruhn, D. (1976) Clin. Chim. Acta 73, 453-461.

12. Thiele, K. G. (1973) Klin. Wochenschr. 51, 339-345.

13. Szasz, G. (1970) Z. Klin. Chem. Klin. Biochem. 8, 1-8.

14. Levy, A. \& Dubach, U. C. (1972) Klin. Wochenschr. 50, 438-441.

15. Werner, M., Maruhn, D. \& Atoba, M. (1969) J. Chromatog. $40,254-263$.

16. Maruhn, D. (1979) Exkretionsmuster von Enzymen im Harn unter physiologischen und pathologischen Bedingungen. Habilitationsschrift, Universität-Gesamthochschule Essen.

17. Pfleiderer, G., Baier, M., Albrecht, W., Mondorf, A. W., Stefanescu, T., Scherberich, J. E. \& Müller, H. (1980) Kidney International $17,242-249$.

18. Dubach, U. C. \& Padlina, G. (1966) Klin. Wochenschr. 44, $180-186$
19. Burchardt, U., Peters, J. E., Neef, L., Thulin, H., Gründig, C. A. \& Haschen, R. J. (1977) Z. Med. Labor.-Diagn. 18, $190-212$.

20. Peters, J. E., Mampel, E., Schneider, I., Burchardt, U., Fukala, E., Ahrens, I. \& Haschen, R. J. (1972) Clin. Chim. Acta 37, 213-224.

21. Baggio, B., Gambaro, G., Ossi, E., Favaro, S. \& Borsatti, A. (1983) J. Urology $129,1161-1162$

22. Dubach, U. C. \& Redinger, R. (1964) Urol. Int. 17, 6583.

23. Amador, E., Zimmermann, T. S. \& Wacker, W. E. C. (1963) J. Am. Med. Ass. 185, 769-775.

24. Hansert, E., Federkiel, H. \& Stamm, D. (1984) J. Clin. Chem. Clin. Biochem. 22, 791-810.

25. Harms, U. (1982) Biomathematik, Statistik und Dokumentation. Harms Verlag, Kiel.

26. Mattenheimer, H. (1974) Methoden der enzymischen Analyse I, S. 64-74 (Bergmeyer, H. U., Hrsg.) Verlag Chemie/ Weinheim, Bergstraße.

27. Scherberich, J. E., Falkenberg, F. W., Mondorf, A. W., Müller, H. \& Pfleiderer, G. (1974) Clin. Chim. Acta 55, 179-197.

28. Mattenheimer, H., Frölke, W., Grötsch, H. \& Simane, Z., Identification of Inhibitors of Urinary Alanine Aminopeptidase, Publikation in Vorbereitung.

Prof. Dr. J. Breuer

Zentral-Labor

Marienhospital

Virchowstraße 135

D-4650 Gelsenkirchen 
